\title{
Effect of meso-Hexestrol, a Synthetic Estrogen, on S-Tubulin
}

\author{
Yumiko Sakakibara, ${ }^{a}$ Taiko Oda, ${ }^{a}$ Aiko Hirata, ${ }^{b}$ Michio Matsuhashi, ${ }^{b}$ and Yoshihiro Sato ${ }^{*, a}$ \\ Biochemistry Division, Kyoritsu College of Pharmacy, ${ }^{a}$ Shibakoen I-chome, Minato-ku, Tokyo 105, Japan and Institute of Applied Microbiology, The \\ University of Tokyo, ${ }^{b}$ Yayoi 1-chome, Bunkyo-ku, Tokyo 113, Japan. Received June 26, 1990
}

\begin{abstract}
We have reported that meso-hexestrol, a synthetic estrogen, inhibits microtubule assembly and induces microtubule proteins into twisted ribbon structures. On the other hand, Serrano et al. proved that S-tubulin, which lacks the C-terminal moiety of tubulin subunits, assembles into sheet structures in the absence of microtubule-associated proteins (MAPs). In the present investigation, we attempted to clarify whether meso-hexestrol could induce the ribbon structure from S-tubulin. meso-Hexestrol delayed the initiation of polymerization of S-tubulin into sheet structures in a dose-dependent manner below $50 \mu \mathrm{M}$. But the effect of meso-hexestrol on S-tubulin was reduced in the presence of either tau or microtubule-associated protein 2 (MAP2) in a MAPs-concentration-dependent manner. At concentrations higher than $100 \mu \mathrm{M}$, meso-hexestrol inhibited the polymerization of S-tubulin into sheet structures, without forming ribbon structures. The present results may indicate that moso-hexestrol interacts with S-tubulin, and its interaction is affected by MAPs.
\end{abstract}

Keywords meso-hexestrol; S-tubulin; microtubule-associated protein; ribbon structure; synthetic estrogen

meso-Hexestrol synthesized by Dodds et al. ${ }^{1)}$ was initially utilized $^{2)}$ as a derivative of diethylstilbestrol (DES), a synthetic estrogen. Later, DES was shown to be a carcinogen in humans ${ }^{3)}$ and experimental animals. ${ }^{4)}$ meso-Hexestrol similarly induced a number of renal carcinoma foci as DES or $17 \beta$-estradiol. ${ }^{5)}$ At present, it is not known whether the oncogenic effects of estrogens are exerted through their hormonal properties or whether they behave as chemical carcinogens. Metzler and McLachlan reported that DES is metabolized to $\beta$-dienestrol $(Z, Z$-dienestrol) by a peroxidase-mediated reaction leading to reactive products and deoxyribonucleic acid (DNA) binding. ${ }^{6}$ There was a good association between the metabolic conversion of DES analogs via a peroxidase-mediated oxidative pathway and their ability to induce cell transformation. ${ }^{7)}$ meso-Hexestrol is not metabolized via this route ${ }^{8)}$ and does not induce significant levels of cell transformation. ${ }^{7)}$

We have reported the inhibition of DES on microtubule assembly in vitro, ${ }^{9)}$ and similar results were successively presented by two research groups. ${ }^{10)}$ Recently, Epe et al. reported that DES binds covalently and selectively to the C-terminal domain of $\beta$-tubulin after peroxidative action in vitro, while meso-hexestrol, which is unable to form quinones by peroxidase-mediate oxidation, fails to bind. ${ }^{11)}$

In a preceding paper, ${ }^{12)}$ we showed that DES analogues can induce the formation of twisted ribbon structures from microtubule proteins, and Chandorille et al. ${ }^{13)}$ also observed similar ribbon structures. Recently, we reported the effects of $(R, R)-(+)-,(S, S)-(-)-, d l$ - and meso-hexestrols on microtubule assembly from a stereochemical point of view. ${ }^{14)}$ We could not form the ribbon structures from PC-tubulin in the presence of meso-hexestrol with $10 \%(\mathrm{v} / \mathrm{v})$ dimethyl sulfoxide (DMSO), but could form them with microtubuleassociated proteins (MAPs). ${ }^{12)}$

The present experiments were designed to clarify whether meso-hexestrol can induce the ribbon structures from S-tubulin, which lacked a C-terminal MAP binding region, ${ }^{15)}$ and to further demonstrate the effect of MAPs on S-tubulin polymerization and the interaction of meso-hexestrol with S-tubulin in the presence of MAPs. It was found that meso-hexestrol inhibited the polymerization of S-tubulin into sheets, but could not induce the ribbon formation from S-tubulin.

\section{Materials and Methods}

Preparation of Microtubule Proteins, Tubulin and MAPs Microtubule proteins were prepared from porcine brains by two cycles of temperature-dependent assembly-disassembly by the method of Shelanski et al. with some modifications. ${ }^{16)}$ The microtubule proteins were stored at $-70^{\circ} \mathrm{C}$ for later use. PC-tubulin was prepared from two-cycle microtubule proteins by the phosphocellulose method described by Kumagai and Nishida. ${ }^{17)}$ Tau and MAP2 were prepared by subjecting MAPs to gel filtration on Ultrogel ACA 34 (LKB) as described by Fellous $e t a l .{ }^{18)}$ The purified tau and MAP2 were dialyzed against PC-buffer ( $0.1 \mathrm{M} 2$-( $N$-morpholino)ethanesulfonic acid, $0.5 \mathrm{~mm} \mathrm{MgCl}_{2}$, $1 \mathrm{~mm} \beta$-mercaptoethanol, $2 \mathrm{~mm}$ ethyleneglycol-bis(2-aminoethylether)$N, N, N^{\prime}, N^{\prime}$-tetraacetic acid, $0.1 \mathrm{~mm}$ ethylenediaminetetraacetic acid (EDTA), $0.5 \mathrm{~mm}$ guanosine triphosphate $(\mathrm{GTP})(\mathrm{pH} 6.8))$ and stored at $-70^{\circ} \mathrm{C}$. Analysis of both tau and MAP2 preparation by sodium dodecylsulfate polyacrylamide gel electrophoresis (SDS-PAGE) showed neither crosscontamination nor contamination by tubulin.

Tubulin Digestion S-Tubulin was prepared by the method of Serrano et al. which performs the digestive removal of the C-terminal regions of both $\alpha$ - and $\beta$-subunits of PC-tubulin. ${ }^{19)}$ PC-Tubulin $(2 \mathrm{mg} / \mathrm{ml})$ in a PC-buffer was digested with $1 \%$ subtilisin for $30 \mathrm{~min}$ at $30^{\circ} \mathrm{C}$. The reaction was stopped by the addition of $1 \mathrm{~mm}$ phenylmethylsulfonyl fluoride, and one polymerization-depolymerization cycle was done by the addition of $1 \mathrm{~mm}$ GTP and incubation of the sample for $30 \mathrm{~min}$ at $37^{\circ} \mathrm{C}$. After these steps, the resulting depolymerized S-tubulin protein in a PC-buffer at $0-2{ }^{\circ} \mathrm{C}$ was characterized by SDS-PAGE and was used immediately for assembly assay.

Electrophoresis SDS-PAGE was performed by the method of Laemmli $^{20)}$ in the case of tau and MAP2. Analysis of PC-tubulin and S-tubulin was carried out on $7.5 \%$ polyacrylamide gel by the method of Best et al. ${ }^{21)}$

Assembly Assay The effect of meso-hexestrol on S-tubulin at $37^{\circ} \mathrm{C}$ was determined by turbidity measurement ${ }^{22)}$ at $400 \mathrm{~nm}$ using a UVIDEC $430 \mathrm{~B}$ double-beam spectrometer equipped with a thermostatically controlled cell holder. Assembly of S-tubulin was performed at a concentration of $1.5 \mathrm{mg}$ protein per $\mathrm{ml}$ of PC-buffer $(15 \mu \mathrm{M}){ }^{23)}$ meso-Hexestrol was dissolved in a $1: 1$ mixture of DMSO and $N, N$-dimethylformamide, ${ }^{24)}$ and this solution was added to the protein solution at a volume ratio of $2 \%$. The reaction was started by shifting up the temperature at $37^{\circ} \mathrm{C}$.

Electron Microscopy Samples were fixed by the addition of 9 volume of a PC-buffer containing 1\% glutaraldehyde. A few minutes later, carbon-coated collodion film on 150 mesh copper grids was placed on drops of a fixed sample solution on a sheet of Parafilm and rinsed with the same buffer. The samples were then negatively stained with $1 \%$ uranyl acetate solution and air-dried. Specimens were examined on a JEOL 200 CX electron microscope at $100 \mathrm{kV}$.

Protein Concentration Concentrations of PC-tubulin, S-tubulin, tau and MAP2 were determined by the method of Lowry et al. ${ }^{25)}$ using bovine 
serum albumin as the standard

Materials meso-Hexestrol ${ }^{26)}$ was obtained from Wako Pure Chemical Industries Ltd. (Osaka) and subtilisin (Carlsberg) (E.C. 3.4.21.14) was from Sigma Chemical Corporation (St. Louis, Mo., U.S.A.). Adenosine triphosphate (ATP) and GTP were obtained from Yamasa Shoyu Co., Ltd. (Choshi), and the materials for electron microscopy were from Nissin EM Co., Ltd. (Tokyo). All other reagents were obtained from Wako Pure Chemical Industries Ltd. (Osaka).

\section{Results and Discussion}

Concentration-Dependence of the Effect of meso-Hexestrol on S-Tubulin We examined the effect of concentrations of meso-hexestrol on S-tubulin polymerization. $\mathrm{S}$-Tubulin was known to assemble into sheet structures in

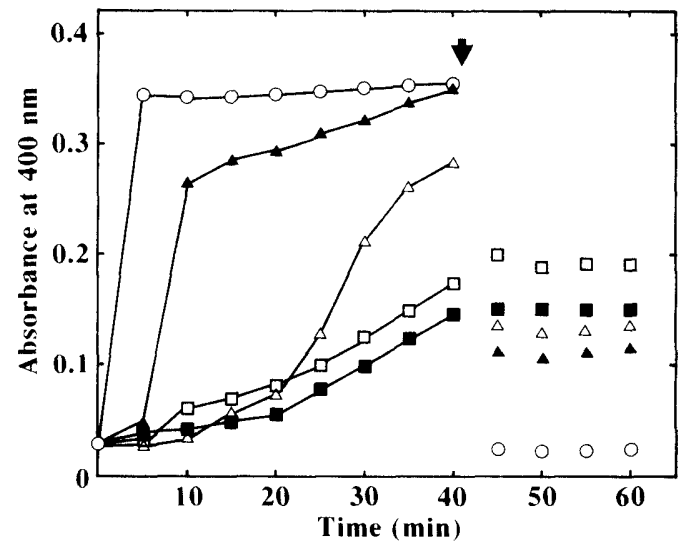

Fig. 1. Turbidimetric Analysis of the Effect of meso-Hexestrol on Polymerization of S-Tubulin

For experimental detail see Materials and Methods. meso-Hexestrol was added to S-tubulin $(1.5 \mathrm{mg} / \mathrm{ml}, 15 \mu \mathrm{M})$ at $0 \mathrm{~min}$. The final concentrations of meso-hexestrol were: $0.0 \mu \mathrm{M}: \Delta, 20 \mu \mathrm{m} ; \triangle, 50 \mu \mathrm{m} ; \square, 100 \mu \mathrm{M} ; \square, 200 \mu \mathrm{m}$. Arrow indicates the temperature shift down to $0^{\circ} \mathrm{C}$
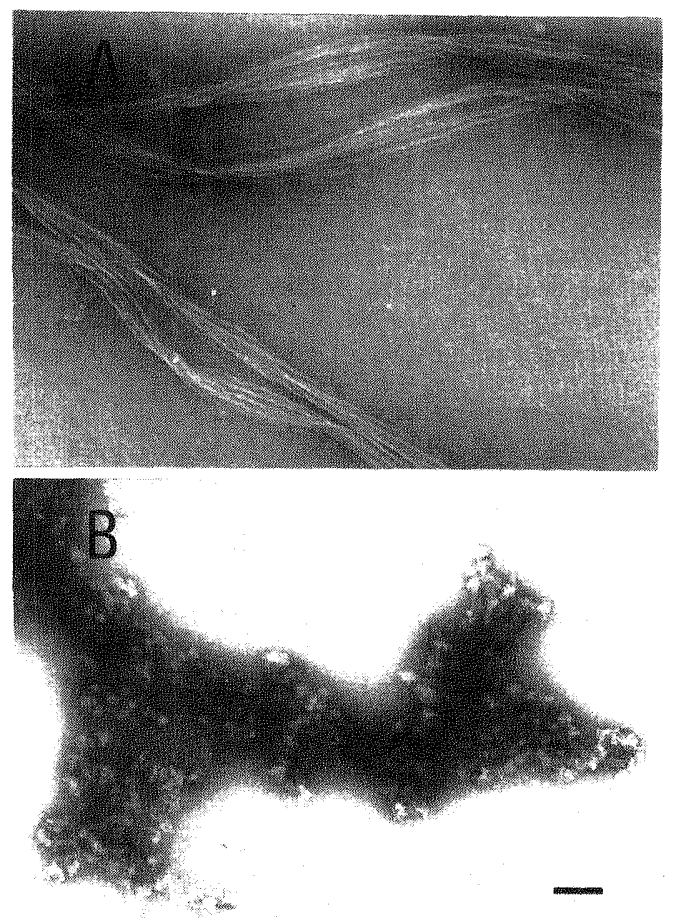

Fig. 2. Electron Micrograph of Sample of S-Tubulin Incubated at $37^{\circ} \mathrm{C}$ for $30 \mathrm{~min}$ in the Absence of meso-Hexestrol (A), and in the Presence of $200 \mu \mathrm{M}$ meso-Hexestrol (B) in Fig. 1

Bar, $100 \mathrm{~nm}$ the absence of MAPs. ${ }^{15 c)}$ meso-Hexestrol inhibited the sheet assembly of S-tubulin in a concentration-dependent manner. As shown in Fig. 1, in the presence of $20 \mu \mathrm{M}$ meso-hexestrol, the turbidity at $10 \mathrm{~min}$ after incubation was suppressed to $70 \%$ of the control, but the final level of the turbidity was attained by prolonged incubation. In the presence of $50 \mu \mathrm{M}$ meso-hexestrol the turbidity increased after $10 \mathrm{~min}$ incubation, and with 100 and $200 \mu \mathrm{M}$ meso-hexestrol, only slow turbidity increases were seen. When the reaction mixture was cooled to $0^{\circ} \mathrm{C}$ after $40 \mathrm{~min}$ of incubation at $37^{\circ} \mathrm{C}$, the turbidity in the control decreased instantly to a low level. However, in experiments in the presence of 20 and $50 \mu \mathrm{M}$ meso-hexestrol, partial turbidity decreases were observed after the temperature shift down to $0^{\circ} \mathrm{C}$. On the other hand, in experiments in the presence of 100 and $200 \mu \mathrm{M}$ meso-hexestrol, no turbidity decrease was seen after the cold treatment at $0{ }^{\circ} \mathrm{C}$. The preparations of S-tubulin incubated for $30 \mathrm{~min}$ were examined with electron microscopy. The control sample in the absence of meso-hexestrol showed long sheet structures (Fig. 2A), while the sample in the presence of $50 \mu \mathrm{M}$ meso-hexestrol showed short sheets. Samples in the presence of meso-hexestrol at concentrations higher than $100 \mu \mathrm{M}$ formed only irregular aggregates (Fig. 2B). Similar aggregates were observed also after the temperature shift down.

Effect of MAPs on S-Tubulin Polymerization The above experiments were performed using S-tubulin in the absence of MAPs. However, in the previous experiment, ${ }^{12}$ ) formation of a ribbon structure was observed from microtubule proteins in the presence of meso-hexestrol but not from a PC-tubulin which was free from MAPs. Moreover, the ribbon formation from PC-tubulin could be demonstrated after addition of MAPs. ${ }^{12)}$ Since mesohexestrol could not induce ribbon formation from S-tubulin in the absence of MAPs, more detailed examinations were performed in the presence of MAPs. Prior to the examination in the presence of meso-hexestrol, the effect of MAPs on S-tubulin polymerization was investigated in its absence. At $0.015 \mu \mathrm{M}$ (MAP/S-tubulin $=1 / 1000(\mathrm{~mol} / \mathrm{mol})$ ), both tau and MAP2 had practically no effect on S-tubulin polymerization as measured by turbidity analysis. In the presence of $1.5 \mu \mathrm{M}$ tau or $0.15-1.5 \mu \mathrm{M} \mathrm{MAP} 2, \mathrm{~S}$-tubulin
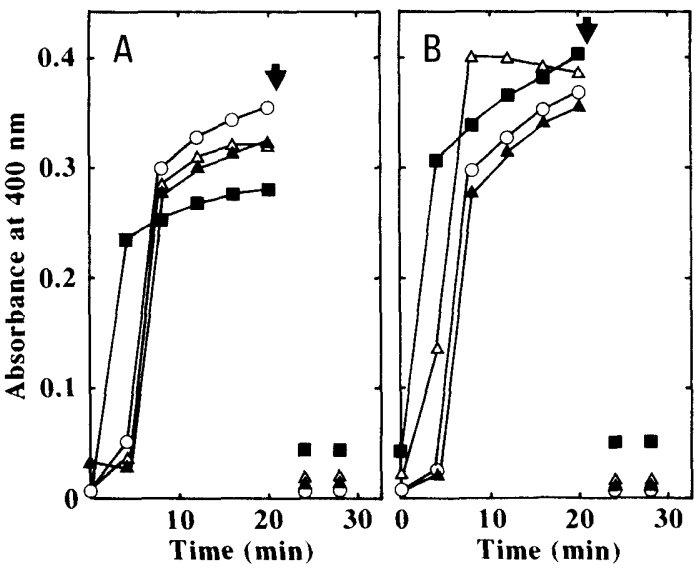

Fig. 3. Turbidimetric Analysis of the S-Tubulin Polymerization in the Presence of Tau (A) or MAP2 (B)

A mixture of S-tubulin $(1.5 \mathrm{mg} / \mathrm{ml}, 15 \mu \mathrm{M})$ and MAP was preincubated in ice for $30 \mathrm{~min}$ and incubated at $37^{\circ} \mathrm{C}$. The final concentrations of MAPs were: $0,0 \mu \mathrm{M}$; $0.015 \mu \mathrm{m} ; \triangle, 0.15 \mu \mathrm{M} ; \mathbf{\square}, 1.5 \mu \mathrm{M}$. Mixtures were then shifted down to $0{ }^{\circ} \mathrm{C}$ at arrow. 
polymerization was initially promoted. But the turbidity after 20 min incubation with $1.5 \mu \mathrm{M}$ tau was lower than the control without tau, whereas in the presence of $0.15-1.5 \mu \mathrm{M}$ MAP2, turbidity increased in a concentration-dependent manner (Fig. 3). When the reaction mixtures were cooled to $0{ }^{\circ} \mathrm{C}$ after $20 \mathrm{~min}$ of incubation at $37^{\circ} \mathrm{C}$, the turbidities in all samples decreased instantly to low levels. Electron microscopy indicated that sheet structures were formed from S-tubulin in all experiments, but in the presence of $1.5 \mu \mathrm{M}$ tau or MAP2, partially microtubule-like structures could be observed as pointed out by arrows (Fig. 4). Serrano et al. reported that $\mathrm{PC}$-tubulin has a MAP binding site in the C-terminal region ${ }^{15 a, b)}$ and that the S-tubulin as-
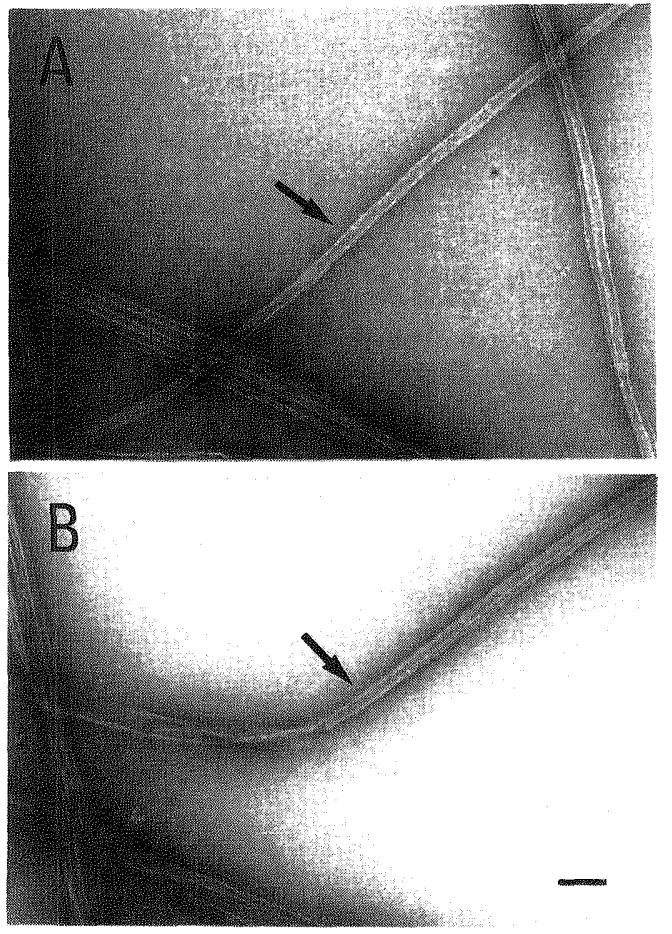

Fig. 4. Electron Micrographs of Samples of S-Tubulin Incubated at $37^{\circ} \mathrm{C}$ for $20 \mathrm{~min}$ in the Presence of $1.5 \mu \mathrm{M}$ Tau (A) or $1.5 \mu \mathrm{M} \mathrm{MAP2}$ (B) in Fig. 3

Arrows show the microtubule-like structures. Bar, $100 \mathrm{~nm}$. sembly has no requirement for MAP2 when analyzed by the amounts of assembled proteins. ${ }^{27)}$ In the present experiment, however, it was shown that both tau and MAP2 have effects on S-tubulin polymerization when analyzed by turbidity and electron microscopy. These results indicate that MAPs interact with $S$-tubulin and change its polymer structures. However, we could not find definite polymer structure differences in the presence of MAPs between tau and MAP2 by electron microscopic analysis.

Effect of meso-Hexestrol on S-Tubulin in the Presence of MAPs Next, we examined the effect of $50 \mu \mathrm{M}$ mesohexestrol on polymerization of $15 \mu \mathrm{M}$ S-tubulin in the presence of MAPs (Fig. 5). At the concentration of $0.015 \mu \mathrm{M}$, both tau and MAP2 had no effect on the inhibition of S-tubulin assembly by meso-hexestrol, but at $0.15-1.5 \mu \mathrm{M}$ tau or MAP2, the inhibitory effect of meso-hexestrol on $S$-tubulin polymerization was significantly reduced. When the reaction mixtures were cooled to $0^{\circ} \mathrm{C}$ at $40 \mathrm{~min}$ of incubation at $37^{\circ} \mathrm{C}$, the turbidities in the control and in the presence of $0.015-0.15 \mu \mathrm{M}$ tau or MAP2 decreased instantly to low levels. Whereas, in experiments in the presence of $1.5 \mu \mathrm{M}$ tau or MAP2, partial turbidity decreases were observed after the temperature shift down to $0^{\circ} \mathrm{C}$. Thus, MAPs reduce the effect of meso-hexestrol on S-tubulin assembly. The following explanations for these results are possible: first, meso-hexestrol may have higher affinities to MAPs than to S-tubulin so MAPs removed the drug from S-tubulin. Secondly, MAPs and meso-hexestrol may have a common interaction-site on S-tubulin which is different from the known MAP binding site at the C-terminal of the tubulin molecule, and MAPs may exclude meso-hexestrol from the interaction-site. Thirdly, MAPs may protect and strengthen the S-tubulin sheet structure.

In addition, at $4.5 \mu \mathrm{M}$ tau $[\mathrm{tau} / \mathrm{S}-\mathrm{tubulin}=3 / 10 \mathrm{(mol} /-$ mol)], some sheet structures were observed even in the presence of $200 \mu \mathrm{M}$ meso-hexestrol (not shown). The result that $\mathrm{S}$-tubulin could not form ribbon structures in the presence of meso-hexestrol suggests that the carboxyterminal domain of tubulin is indispensable to mesohexestrol-induced ribbon formation. On the other hand, Serrano et al. found that in the presence of vinblastine,

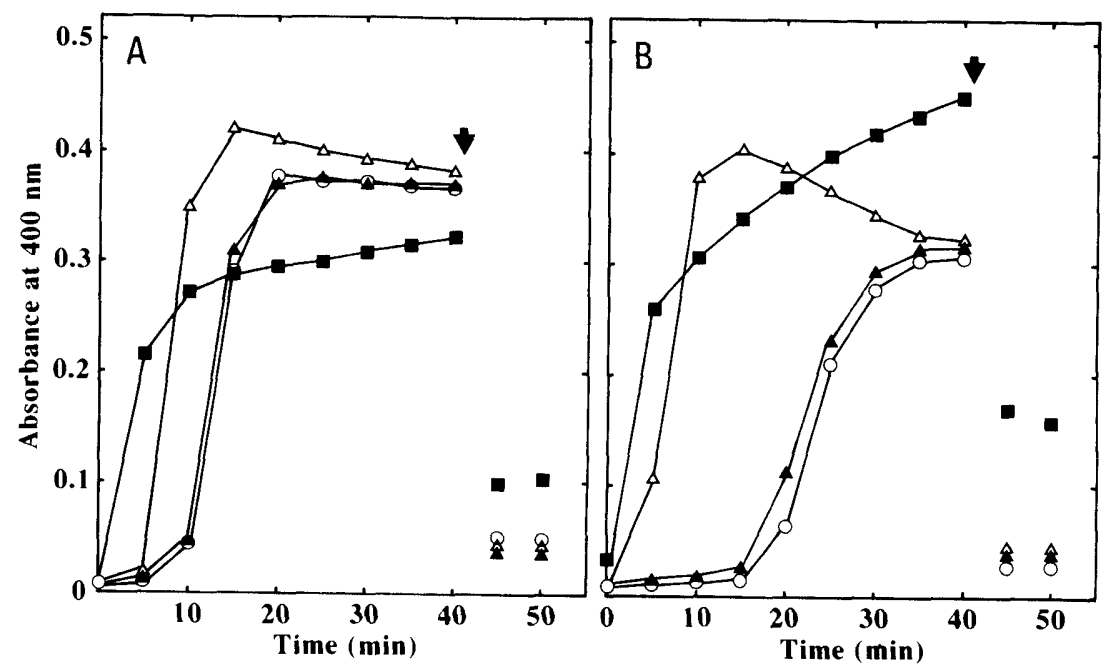

Fig. 5. Turbidimetric Analysis of the Effect of $50 \mu \mathrm{M}$ meso-Hexestrol on Polymerization of S-Tubulin in the Presence of Tau (A) or MAP2 (B)

S-Tubulin $(1.5 \mathrm{mg} / \mathrm{ml}, 15 \mu \mathrm{M})$ and MAPs were preincubated in ice for $30 \mathrm{~min}$. meso-Hexestrol was added to the S-tubulin solution at 0 min, and the reaction was started by transfer to $37^{\circ} \mathrm{C}$. The final concentration of MAPs was: $0,0 \mu \mathrm{M} ; \boldsymbol{\Delta}, 0.015 \mu \mathrm{M} ; \Delta .0 .15 \mu \mathrm{M} ;$ and $\mathbf{\square}, 1.5 \mu \mathrm{M}$. Arrow indicates the temperature shift down
to $0^{\circ} \mathrm{C}$. 
S-tubulin polymerized into spiral structures in the absence of MAPs. ${ }^{19)}$ Therefore, the present results show that the meso-hexestrol-induced ribbons contrast with the vinblastine-induced spirals.

The present study showed that S-tubulin could not form ribbons in the presence of meso-hexestrol, suggesting that the C-terminal regions of PC-tubulin contribute to the formation of ribbon structures in the presence of mesohexestrol.

Acknowledgment This study was supported in part by a Grant-in-Aid for Scientific Research (No. 61571064) from the Ministry of Education, Science and Culture of Japan; and by the Science Resarch Promotion Fund from the Japan Private School Promotion Foundation. We thank Misses R. En-moto, A. Kosugi, M. Nomura, Y. Ando, Y. Miyazaki for their technical assistance.

\section{References and Notes}

1) E. C. Dodds, L. Golberg, W. Lawson, and R. Robinson, Proc. $R$. Soc. London, Ser. B, 127, 140 (1939).

2) S. W. Landvatter and J. A. Katzenellenbogen, Mol. Pharmacol,, 20 43 (1981).

3) A. L. Herbst, H. Ulfelder, and D. C. Poskanzer, N. Engl. J. Med., 284, 878 (1971); G. Krishna, G. U. Corsini, J. Gillette, and B. B. Brodie, Toxicol. Appl. Pharmacol., 23, 794 (1972).

4) H. Kirkman and R. L. Bacon, Cancer Res., 10, 122 (1950).

5) J. J. Li, S. A. Li, J. K. Klicka, J. A. Parsons, and L. K. T. Lam, Cancer Res., 43, 5200 (1983).

6) M. Metzler and J. A. McLachlan, Biochem. Biophys. Res. Commun., 85, 874 (1978).

7) J. A. McLachlan, A. Wong, G. H. Degen, and J. C. Barrett, Cancer Res., 42, 3040 (1982).

8) M. Metzler, R. Gottschlich, and J. A. McLachlan, "Estrogens in the Environment," ed. by J. A. McLachlan, Elsevier-North Holland Biomedical Press, Inc., Amsterdam, 1980, pp. 293-303.

9) Y. Sato, T. Murai, M. Tsumuraya, H. Saitô, and M. Kodama, Gann, 75, 1046 (1984).
10) D. C. Sharp and J. M. Parry, Carcinogenesis, 6, 865 (1985); B. Hartley-Asp, J. Deinum, and M. Wallin, Mutat. Res., 143, 231 (1985).

11) B. Epe, J. Hegler, and M. Metzler, Carcinogenesis, 8, 1271 (1985).

12) Y. Sato, T. Murai, T. Oda, H. Saitô, M. Kodama, and A. Hirata, J. Biochem. (Tokyo), 101, 1247 (1987).

13) M. M. Chaudoreille, V. Peyrot, D. Braguer, and A. Crevat, Mol. Pharmacol., 32, 731 (1987)

14) Y. Sakakibara, K. Hasegawa, T. Oda, H. Saitô, M. Kodama, A. Hirata, M. Mastuhashi, and Y. Sato, Biochem. Pharmacol., 39, 167 (1990).

15) a) L. Serrano, J. Avila, and R. B. Maccioni, Biochemistry, 23, 4675 (1984); b) L. Serrano, E. Montejo de Garcini, M. A. Hernandez, and J. Avila, Eur. J. Biochem., 153, 595 (1985); c) L. Serrano, J. de la Torre, R. B. Maccioni, and J. Avila, Proc. Natl. Acad. Sci. U.S.A., 81, 5989 (1984).

16) M. L. Shelanski, F. Gaskin, and C. R. Cantor, Proc. Natl. Acad. Sci. U.S.A., 70, 765 (1973); M. Ishikawa, H. Murofushi, and H. Sakai, J. Biochem. (Tokyo), 94, 1209 (1983).

17) H. Kumagai and E. Nishida, J. Biochem. (Tokyo), 85, 1267 (1979).

18) A. Fellous, J. Francon, A.-M. Lennon, and J. Nunez, Eur. J. Biochem., 78, 167 (1977).

19) L. Serrano, J. de la Torre, R. F. Luduena, and J. Avila, Arch. Biochem. Biophys., 249, 611 (1986).

20) U. K. Laemmli, Nature (London), 227, 680 (1970).

21) D. Best, P. J. Warr, and K. Gull, Anal. Biochem., 114, 281 (1981).

22) F. Gaskin, C. R. Cantor, and M. L. Shelanski, J. Mol. Biol., 89, 737 (1974).

23) Molar concentrations shown in this paper are based on the following molecular weights: S-tubulin, 100000; tau, 58000; and MAP2, 271000.

24) Y. Sato, Y. Saito, Y. Shiratori, S. Shoda, and J. Hosoi, Nippon Kagaku Kaishi, 1981, 746.

25) O. H. Lowry, N. J. Rosebrough, A. L. Farr, and R. J. Randall, J. Biol. Chem., 193, 265 (1951).

26) T. Oda, T. Murai, and Y. Sato, Chem. Pharm. Bull., 36, 1534 (1988); H. Saitô, M. Yokoi, M. Aida, M. Kodama, T. Oda, and Y. Sato, Magn. Reson. Chem., 26, 155 (1988).

27) R. B. Maccioni, L. Serrano, J. Avila, and J. R. Cann, Eur. J. Biochem., 156, 375 (1986). 